\title{
Downregulation of chloroplast protease AtDeg5 leads to changes in chronological progression of ontogenetic stages, leaf morphology and chloroplast ultrastructure in Arabidopsis
}

\author{
Małgorzata Baranek ${ }^{1 *}$, Tomasz P. Wyka², Grzegorz Jackowski ${ }^{1}$ \\ 'Department of Plant Physiology, Institute of Experimental Biology, Faculty of Biology, Adam Mickiewicz University, Umultowska 89, 61-614 Poznań, Poland \\ 2 Department of General Botany, Institute of Experimental Biology, Faculty of Biology, Adam Mickiewicz University, Umultowska 89, 61-614 Poznań, Poland
}

\section{Abstract}

The chloroplast protein AtDeg5 is a serine-type protease peripherally attached to thylakoid membrane at its lumenal side. Since reliable data regarding the role of AtDeg 5 in controlling the course of growth and developmental processes are extremely limited, two independent T-DNA insertional lines with different extent of AtDeg5 reduction were prepared and ontogenesis stage-based analysis performed. Both mutant lines displayed a compensatory overaccumulation of AtDeg8. The repression of AtDeg5 protease altered a range of phenotypic features in at least one of the mutants, with the most prominent being changes in chronological progression of development and growth of individual rosette leaves, flower production and silique ripening as well as in the area of fully expanded leaves and chloroplast ultrastructure. By analyzing the results of parallel-mutant screening we conclude that AtDeg8 overdose may rescue 23\% of AtDeg5 deficiency with regard to some AtDeg5-controlled traits; alternatively AtDeg5 may have catalytic sites in excess so that these traits might remain unaltered when AtDeg5 pool is reduced by 23\%. For some other AtDeg5-dependent traits the absence of excessive amount of AtDeg5 catalytic sites, lack of AtDeg5 dosage effect and inability of AtDeg8 to compensate deficiency or absence of AtDeg5 occurred.

Keywords: AtDeg5 protease; chronological progression; leaf morphology; chloroplast ultrastructure; starch grains

\section{Introduction}

The results of studies performed in recent years show that regulatory protein hydrolysis, catalyzed by proteolytic enzymes is directly or indirectly involved in a majority of cellular processes of all living organisms including plants and it is thus not surprising that the MEROPS database (9.10 release) lists as many as 783 known and putative proteases in Arabidopsis thaliana, representing about $3 \%$ of all proteins identified in this taxon. Proteolysis is considered a vital factor influencing whole-plant maintenance, structure and functions under changing environmental conditions and in changing ontogenetical context [1]. It is widely believed that proteases are involved in protein quality control and protein turnover processes. The protein quality control comprises a hydrolysis of proteins which have been damaged due to mutations or exposition of plants to stressing environmental conditions, synthesized at redundant quantity

\footnotetext{
*Corresponding author. Email: gosiab@amu.edu.pl
}

Handling Editor: Beata Zagórska-Marek or sorted to incorrect cell compartment. Protein turnover, in its turn comprises a hydrolysis of proteins which become unnecessary in a defined spatio-temporal context under non-stressing conditions.

Deg proteases were first discovered and described in late 1980s by studying Escherichia coli mutants unable to grow at elevated temperatures $\left(>37^{\circ} \mathrm{C}\right)$ due to their inability to dispose of periplasmic proteins damaged under heat shock conditions [2,3]. In fact the quality of $E$. coli periplasmic proteins is controlled by three Deg proteins namely DegP, DegQ and DegS whose structure and function have been solved in much detail [4-6]. Later Deg proteases were found to occur ubiquitously in cells of all domains of life, from Bacteria through Archea to Eukarya [7]. Sixteen genes coding for proteins orthologous to DegP, Q and S have been identified in the $A$. thaliana nuclear genome and designated AtDEG1-16 [8]. It has been experimentally proven that five of the genes (AtDEG1, 2, 5, 7 and 8 ) encode proteins targeted to chloroplasts, one (AtDEG10) encodes a mitochondriontargeted protein and another one (AtDEG15) a peroxisomal protein. The remaining nine AtDEG genes code for proteins which have been predicted in silico to be targeted either to 
mitochondria or chloroplasts or their localization has not been predicted in silico yet (for a review see [9]).

It was demonstrated that a linear structure of chloroplasttargeted AtDeg proteases involves one or two protease domains (if the second protease domain is present - it is a catalytically inactive version [10]) and 0 to 4 of PDZ domains which are thought to mediate formation of proteolytically active oligomeric forms [11] and select proper substrates [12]. In fact, several lines of evidence show that chloroplasttargeted AtDeg proteases exist as both homo- and heterooligomeric assemblies with trimer as a basic structural unit. For instance, a catalytically active AtDeg1 particle was demonstrated to be a hexamer of a "sealed-cage" type, consisting of two trimeric rings stacked upon each other in such a way that they form an inner, catalytic chamber. A hexamer is stabilized through interactions of protease and PDZ domains originating from adjacent monomers of opposing trimers [11]. In turn AtDeg5 forms trimers in which individual monomers are associated through hydrophobic interactions, hydrogen bonds and $\pi$-cation interaction [13]. AtDeg5 and AtDeg8 have been suggested to make assemblies mediated by association of equimolar amounts of homooligomers of both molecules [14].

In spite of a significant progress which has been made with regard to understanding structural organization of chloroplast-targeted AtDeg oligomers the knowledge of their functions is extremely limited both in terms of identity of chloroplast proteins representing native substrates for AtDeg proteases under stressing or non-stressing conditions and in terms of their role in controlling the course of growth and developmental processes at various levels of plant body organization. Specifically, it was demonstrated for AtDeg1, 7 and 8 that recombinant versions of these enzymes catalyze in vitro degradation of photodamaged PSII reaction center protein PsbA (D1) which in vivo is inherent to PSII repair cycle. The results have suggested that the photodamaged PsbA protein is cleaved in vivo by introduction of multiple cuts by cooperating AtDegs 1, 5/8, 7 and probably AtDeg2 as well [12,14-17]. Similarly, recombinant version of AtDeg1 and AtDeg7 has been demonstrated to be responsible for in vitro degradation of PsbD, PsbS, Lhcb4 and cytb6 as well as PsbB-D, respectively under photoinhibitory conditions $[12,18]$. Later it was demonstrated in a more direct way that Lhcb6 and PsbF apoproteins are degraded in a stress-related manner by AtDeg2 and AtDeg5, respectively [16,19]. Reliable data on the role of chloroplast targeted-AtDegs in controlling the course of growth and developmental processes at various levels of the plant body plan are very scarce - in two separate studies using 4-weeks-old long day grown wild type (WT) plants and mutants devoid of AtDeg2 and AtDeg5 we have suggested recently that AtDeg2 and AtDeg5 have some functions in controlling morphology and anatomy of leaves as well as the chloroplast life cycle under non-stressing conditions $[16,19]$.

To gain a deeper insight into the functional significance of AtDeg chloroplast proteases, and AtDeg5 in particular, we investigated AtDeg5 involvement in plant growth and development by a large-scale, comparative phenotypic screen of WT plants and two deg5 mutants deficient in AtDeg5 protease when growing under non-stressing conditions.
As the extent of the AtDeg5 reduction varied between 23\% (deg5-2) and 100\% (deg5-1) in this way we could study the importance of AtDeg5 protein dosage effect on phenotypic traits. We demonstrate here that AtDeg5 is needed for normal plant ontogenesis since AtDeg5 repression produces significant changes in chronological progression of some growth and development stages, leaf morphology, chloroplast ultrastructure and diurnal transitory starch metabolism. Our results show that AtDeg5 deficiency/absence leads to a compensatory overaccumulation of AtDeg8 - this protease may be functionally interchangeable with AtDeg5, possibly with regard to expression of some phenotypic alterations or AtDeg 5 has enough catalytic sites in excess to function normally even when its pool is lowered to $77 \%$ of the WT plants value. In the case of other phenotypic traits which are altered in $\operatorname{deg} 5$ mutants AtDeg8 is unable to compensate AtDeg5 deficiency or absence.

\section{Material and methods}

\section{Plant material and growth conditions}

Seeds of WT and mutant plants were sown on $1 / 2 \mathrm{Mu}-$ rashige and Skoog [20] medium with agar (for early analyses) or $42 \mathrm{~mm}$ Jiffy peat pellets on sphagnum peat moss and wood pulp (AgroWit, Przylep, Poland; for soil-based analysis) and grown in a growth chamber (NEMA, Netzschkau, Germany) under long day conditions ( $16 \mathrm{~h} \mathrm{:} 8 \mathrm{~h}$, light : dark), with the irradiance set at $110 \mu \mathrm{mol}$ quanta $\mathrm{m}^{-2} \mathrm{~s}^{-1}$, at a $22^{\circ} \mathrm{C}$ (day) $/ 18^{\circ} \mathrm{C}$ (night) temperature cycle and $65 \%$ relative humidity. Seeds of Arabidopsis thaliana insertion lines SAIL_645_D07 (deg5-2 mutant) and SALK_099162 (deg5-1 mutant) were obtained from Nottingham Arabidopsis Stock Center (NASC). T-DNA insertions in DEG5 gene (At4g18370) were confirmed and hetero or homozygosity was analyzed by PCR using the following primers for DEG5 sequence: forward 5'- GAGGATGAAGAAGAGAGAAATG-3' and reverse 5'-CCCAACCCACTTACTACCTAC-3' for deg5-2 mutant, forward 5'-GCTTTTTCCTCAATCTTCAATAC-3' and reverse: 5'-AGGATTTAGTTCACGTCCCTC-3' for deg5-1 mutant, and LBb 5'-GCGTGGACCGCTTGCTGCAACT-3' for the insertion.

\section{Preparation of thylakoids samples}

Intact chloroplasts were prepared from leaves of WT or mutant plants using centrifugation on Percoll gradient, essentially as described in [21]. Thylakoids were isolated by resuspending the pellet of intact chloroplasts in water, centrifuging the suspension for $10 \mathrm{~min}$ at $12500 \mathrm{~g}$ and collected as a green pellet, finally suspended in $10 \%$ glycerol and stored frozen at $-18^{\circ} \mathrm{C}$.

\section{SDS-PAGE, immunoblotting and protein quantitation}

Thylakoid samples were resolved by SDS-PAGE [22] on the basis of equal amount of chlorophyll, electrotransferred onto PVDF membranes and immunostained with primary antibodies against Deg1, Deg2, Deg5, Deg8 and PsbC apoproteins. The immunostaining and the quantification of immunostained bands were performed essentially as described by us previously [23]. 


\section{Chronological progression of selected principal and secondary} stages of growth and development of WT and mutant plants

Chronological progression of seed germination (principal stage $0,[24]$ ) was analyzed by light microscopy (observations performed two times a day) of seeds growing on agar plates. The secondary stages i.e. the time needed to reach seed imbibition, radicle emergence and hypocotyl/cotyledon emergence were determined.

Chronological progression of leaf development (principal stage 1, [24]) was analyzed by visual inspection (performed two times a day) of seedlings growing on Jiffy peat pellets. The secondary stages i.e. the time when cotyledons were fully opened, leaves 1 and 2 and all remaining leaves (3-9) became longer than $1 \mathrm{~mm}$, were determined.

Chronological progression of leaf growth (principal stage 3 defined in a modified way, see "Results" for details) was evaluated by light microscopy (the data were collected once a day). The secondary stages were determined for each leaf individually as the time needed to reach $10 \%, 50 \%$ and $100 \%$ of its final size.

Progression of inflorescence emergence (principal stage $5,[24]$ ) was analyzed by visual inspection (performed two times a day). The only secondary stage, i.e. the time when the first flower bud became longer than $1 \mathrm{~mm}$, had been determined.

Chronological progression of flower production and silique ripening (principal stages 6 and 8, respectively, [24]) were determined by visual inspection (performed two times a day). The secondary stages of the principal stage 6 were determined as the time when the first flower as well as $10 \%$, $50 \%$ and $100 \%$ of flowers opened and the secondary stages of the principal stage 8 as the time when the first silique as well as $10 \%, 50 \%$ and $100 \%$ of the siliques became shattered.

\section{Microscopy}

Whole leaves were digitally photographed using a SteReo Lumar V12 stereomicroscope (Carl Zeiss, Jena, Germany) at $6.4 \times$ magnification, and the images were analyzed with AxioVision Rel 4.8 (Carl Zeiss) and leaf length, width, area, perimeter and shape coefficient (defined according to [25]) were determined.

Transmission electron microscopy of the leaves (JEM 1200 EXII, JEOL, Tokyo, Japan), the negative scanning and digital image analysis were performed essentially as described by us earlier [16].

Scanning electron microscopy of the seeds (EVO 40, Carl Zeiss Microscopy GMBH, Jena, Germany) was performed according to [26], the negative scanning and digital image analysis was done essentially as described in [16].

\section{Starch quantification}

Leaves of WT and mutant plants were harvested at various time points during the day, night and an extended night. The leaves were frozen in liquid nitrogen and soluble carbohydrates were extracted by placing samples into boiling $80 \%$ ethanol and incubating in $80 \%$ ethanol for $18-20 \mathrm{~h}$ at $37^{\circ} \mathrm{C}$. The ethanol fraction was removed by centrifugation $\left(10 \mathrm{~min}\right.$ at $8000 \mathrm{~g}$ at $4^{\circ} \mathrm{C}$ ) and the dried pellet was homogenized in $0.2 \mathrm{~N} \mathrm{KOH}$ and boiled for $1.5 \mathrm{~h}$. Then the mixture was acidified to $\mathrm{pH}$ 5.0, incubated with amyloglucosidase
(10 U) for $24 \mathrm{~h}$ at $55^{\circ} \mathrm{C}$, centrifuged (for $3 \mathrm{~min}$ at $8000 \mathrm{~g}$ ) and the glucose was measured enzymatically as described in [27]. The amount of glucose was calculated against a glucose standard. To determine starch the glucose amount was multiplied by 0.9 coefficient.

\section{Gas exchange analysis}

Determination of $A / C_{i}$ responses was performed using the Li-6400 gas exchange system equipped with the standard $6 \mathrm{~cm}^{2}$ chamber with a built-in red-and-blue LED light source (Li-Cor Inc., Lincoln, NE, USA). For about $60 \mathrm{~min}$ prior to the measurement plants were exposed to $500 \mu \mathrm{mol}$ quanta $\mathrm{m}^{-2} \mathrm{~s}^{-1}$ and the measurements were conducted at $1500 \mu \mathrm{mol}$ quanta $\mathrm{m}^{-2} \mathrm{~s}^{-1}$ while leaf temperature was maintained at $22^{\circ} \mathrm{C}$ and vapor pressure deficit in the chamber did not exceed 1.4 MPa. To generate $A / C_{i}$ response curves, photosynthetic rate $(A)$ was measured at $400,300,200,100,75,50 \mu \mathrm{mol}$ $\mathrm{mol}^{-1} \mathrm{CO}_{2}$, followed by restoration of initial photosynthetic rate at $400 \mu \mathrm{mol} \mathrm{mol}{ }^{-1} \mathrm{CO}_{2}$. Further measurements were done at 500, 750, 1000, 1500 and $1800 \mu \mathrm{mol} \mathrm{mol}^{-1} \mathrm{CO}_{2}$. Values of $A$ were corrected for diffusional leaks as determined with an empty chamber using the same range of $\mathrm{CO}_{2}$ concentrations.

Leaf areas were determined using a flatbed scanner and an image processing software. Since leaves were smaller than the chamber area and varied in size and shape, leaf boundary layer conductances were determined individually for each leaf. Leaves were photocopied, leaf silhouettes were cut out of filter paper, moistened and steady-state evaporation was measured. Boundary layer conductance was computed using the Li-6400 routine. Internal concentrations of $\mathrm{CO}_{2}$ $\left(C_{i}\right)$ were calculated using equations provided in the $\mathrm{Li}-6400$ manual (Li-Cor 2005).

Equations of Farquhar et al. [28] two-phase model were fitted to the $A / C_{i}$ data, with measurements at $C_{i}<400 \mu \mathrm{mol}$ $\mathrm{mol}^{-1} \mathrm{CO}_{2}$ considered to be limited by Rubisco amount, kinetics and activity whereas those at $C_{i}>400 \mu \mathrm{mol} \mathrm{mol}^{-1}$ $\mathrm{CO}_{2}$ to be limited by electron transport used in the regeneration of ribulose 1,5-bisphosphate (RuBP) [29]. Fitting was done with the maximum-likelihood based curve fitting routine of SigmaPlot 11 (Systat Software, San Jose, CA, USA) using kinetic parameters of Rubisco given in [30]. Maximal carboxylation rate $\mathrm{Vc}$, max and maximal electron transport rate Jmax were obtained as parameters in the best fit models. Both $V_{c, \max }$ and $J_{\max }$ were normalized to $25^{\circ} \mathrm{C}$ values using equations given in [31].

\section{Chlorophyll determination}

Chlorophyll concentration was assayed spectrophotometrically according to [32].

\section{Statistical analysis}

Statistical significance of differences in measured parameters were analyzed according to one-way analysis of variance (ANOVA) and Tukey-Kramer multiple comparison test. Means were regarded as significantly different at $P<0.01$. 


\section{Results}

\section{T-DNA insertion mutants for AtDEG5}

To study AtDeg 5 functions seeds of the insertion SAIL_645_D07 line (deg5-2) were obtained from the Nottingham Arabidopsis Stock Centre. For comparison our study also included SALK_099162 line (deg5-1) which was studied previously $[14,19]$. For deg5-2 a confirmed position of the T-DNA insertion is in the 4th intron 872 bp downstream of ATG, whereas the deg5-1 line contains an insertion in the 2nd exon of At4g18370 gene 429 bp downstream of ATG codon (Fig. 1a). Homozygous mutants of both lines were identified in F2 progeny by PCR-based genotyping (Fig. 1b). In accordance with earlier studies [19] the deg5-1 mutant was found to be entirely depleted of AtDeg5 protein, whereas deg5-2 plants showed a modest (but significant) reduction of AtDeg5 level, to $77 \%$ of the value found in WT plants (Fig. 2). No changes in the amount of AtDeg1 and AtDeg2 proteins have been detected in both mutants vs. WT plants. Interestingly, the amount of AtDeg8 increased about 2-fold in the mutant lines (Fig. 2).

\section{Chronological progression of ontogenetic stages in WT and deg5 mutants}

As a first step in performing comparative phenotypic screens a chronological progression of selected stages covering the plant ontogenesis from seed imbibition to fruit ripening has been studied for homozygous deg5-2 and deg5-1 mutants as well as WT plants growing under a 16/8-h photoperiod. The study involved five principal stages defined essentially according to $\mathrm{BBCH}$ scale [33] as adapted for Arabidopsis phenotypes [24], namely: 0 - seed germination, 1 - leaf development, 5 - inflorescence emergence, 6 - flower production and 8 - silique ripening and their secondary stages. In the case of the principal stage 3 an altered $\mathrm{BBCH}$ based definition was developed by us allowing to recognize growth of individual leaves - instead of rosette growth - by introducing numbers indicating accomplishment of various percentages of final sizes by individual leaves (= completion of various secondary stages). Thus the secondary stages of the principal stage 3 are coded by three digits representing the principal stage, the secondary stage and the leaf number, respectively (e.g. 3.45 means the moment when the secondary stage 4 of the principal stage 3 was reached by leaf 5). Fig. 3 shows the time (in days) required to complete individual secondary stages of growth and development by WT plants and the mutants. No significant alterations in chronological progression of secondary ontogenesis stages were observed for deg5-2. In contrast, a variety of WT/deg5-1 significant differences were seen involving progression of secondary stages of principal stages 1, 3,6 and 8. Rosette leaves 1 and 2 as well as 5 of $\operatorname{deg} 5-1$ mutant appeared (secondary stages 1.2 and 1.5) before and after respective leaves of WT plants, indicating that the mutant produced these leaves at an altered rate. The majority of rosette leaves of $\operatorname{deg} 5-1$ mutant (i.e. the leaves 1, 3, 4, 5, 6 and 7) reached their final size 2-6 days later than WT plants, for instance the leaf 1 accomplished its final size (secondary stage 3.91) five days later than the same leaf of WT plant. The remaining leaves of the mutant $(2,8$ and 9) were delayed in comparison to leaves of WT plants (a)
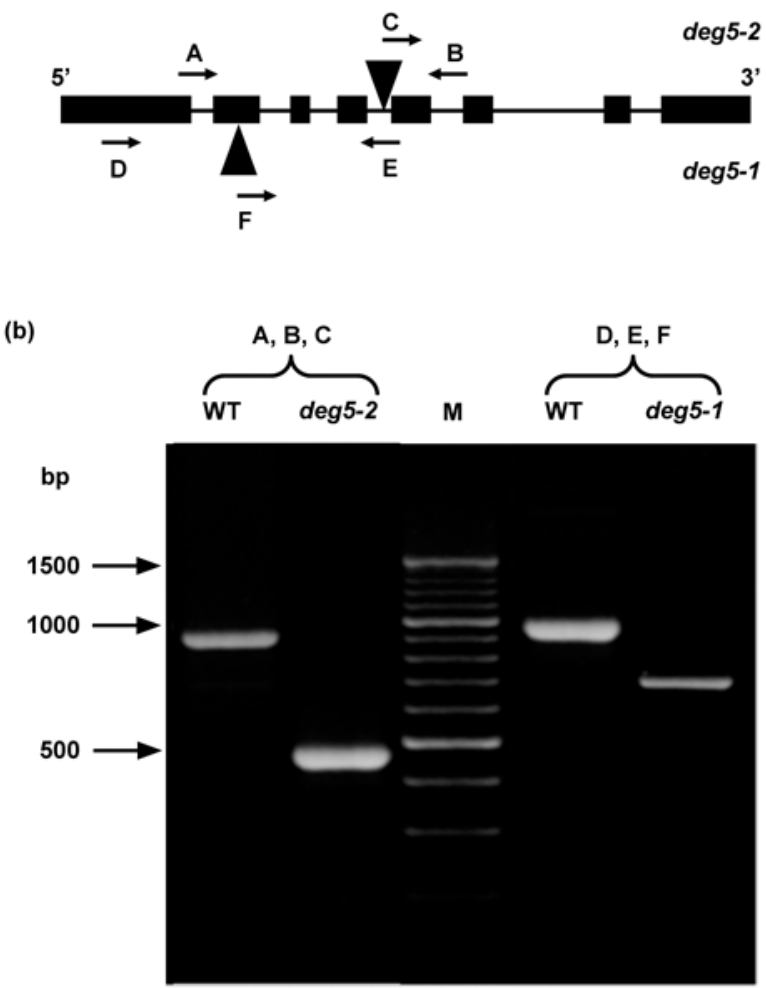

Fig. 1 Identification of deg5 mutants. a Schematic diagram of genomic AtDEG5 gene. Black boxes represent exons, thin lines introns and triangle - site of T-DNA insertion. Arrows indicate the annealing sites of primers used for PCR analysis of genomic DNA. b Confirmation of homozygosity of deg5-2 and deg5-1 mutants by electrophoretic separation of PCR products of genomic DNA of WT and mutant plants. Amplification was performed with primers A, B, C, D, E and F according to the descriptions in Fig. 1a.

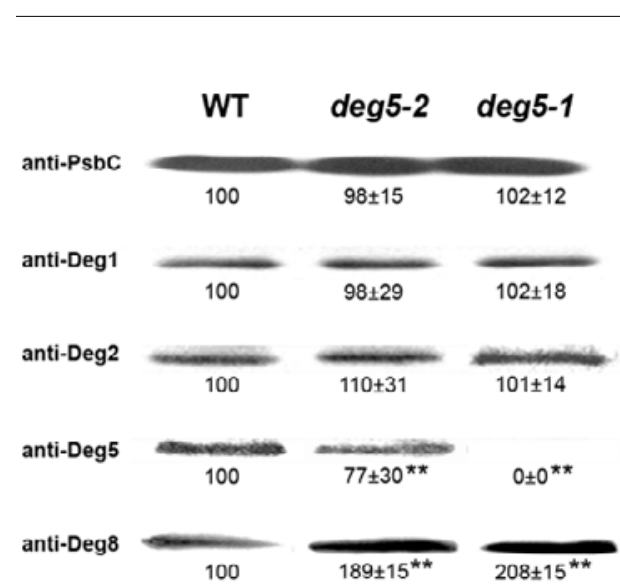

Fig. 2 Immunoblot analysis of levels of chloroplast AtDeg proteins in leaves of WT plants and deg5 mutants. Samples of the thylakoids isolated from leaves of WT and deg5-2 and deg5-1 mutant plants which reached secondary stage 6.0 were subjected to immunoblot analysis with anti-Deg1, Deg2, Deg5, Deg8 and PsbC antibodies. Individual signals of AtDeg proteases or PsbC apoprotein in mutant thylakoids were quantified relative to signals identified for thylakoids of WT plants (100\%) using Gelix One software (Biostep, Jahnsdorf, Germany). Signal strength $\pm S D$ is indicated below the corresponding lanes. The signals were determined by scanning six blots representing two biological replicates. Asterisks indicate the data for which mutant/WT plants differences were significant $(P<0.01)$. 


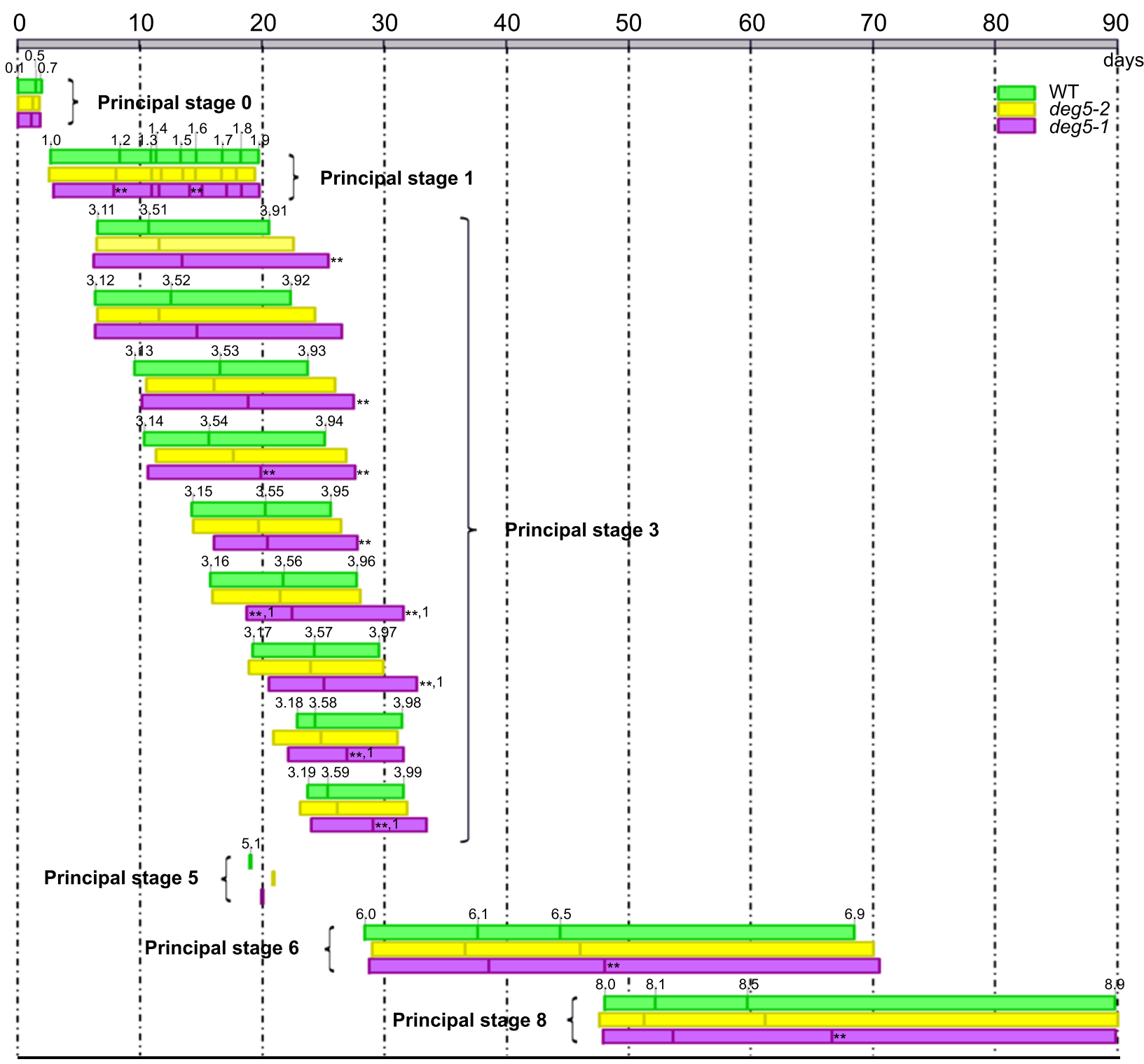

Fig. 3 Analysis of the chronological progression of the principal stages $0,1,3,5,6$ and 8 of plant ontogenesis in WT and deg5 mutant plants. Horizontal, colored boxes indicate the time (in days) which is necessary for WT and deg5-2 and deg5-1 mutant plants to complete individual secondary stages (marked as small vertical bars within colored boxes). Numbers above the colored boxes correspond to secondary stages of ontogenesis. The asterisks indicate the data for which mutant/WT plants differences were significant $(P<0.01)$ and 1 indicate the values for which deg5-1/deg5-2 differences were significant $(P<0.01)$.

as well but the delay was not significant. Furthermore, some leaves of the deg5-1 mutant (No. 4, 8 and 9) were delayed transiently at the moment when they reached $50 \%$ of their final sizes. A delay in growth rate of the majority of leaves of the deg5-1 mutant was accompanied by a modest delay in flower production and silique ripening - as a result $50 \%$ of the flowers opened (secondary stage 6.5) and $50 \%$ of the siliques shattered (secondary stage 8.5 ) significantly later than in WT plants.

\section{Morphology of fully expanded leaves, ultrastructure and diurnal metabolism of transitory starch of chloroplasts of fully expanded leaves of WT and mutant plants}

To investigate the effect of the delay in growth exhibited by the majority of leaves of deg 5 mutants on final leaf morphology digital images of the leaves of both mutants and WT plants were collected and analyzed at secondary stage 6.0, i.e. the moment when the first flower was opened [24]. The leaves 3 and 5 were selected for these analyses since they represent juvenile and mature leaf populations, respectively [34] and AtDeg5 deficiency could have different impact on their morphological features (both leaves became fully expanded at or before secondary stage 6.0 both in mutants and WT plants). The morphological properties of deg5-2 mutant leaves were found to be unchanged relative to those of WT plants whereas deg5-1 mutant plants exhibited a considerable increase in the area and perimeter of both juvenile and mature leaves relative to WT plants and this was due to relevant increases in the leaf length and width (Fig. 4). Since the increases in length and width of the leaf 
blades were roughly proportional leaf shape coefficients remained unaffected. No statistically significant deg5-1/ deg5-2 differences with respect to morphological features of either juvenile or mature leaves were identified.
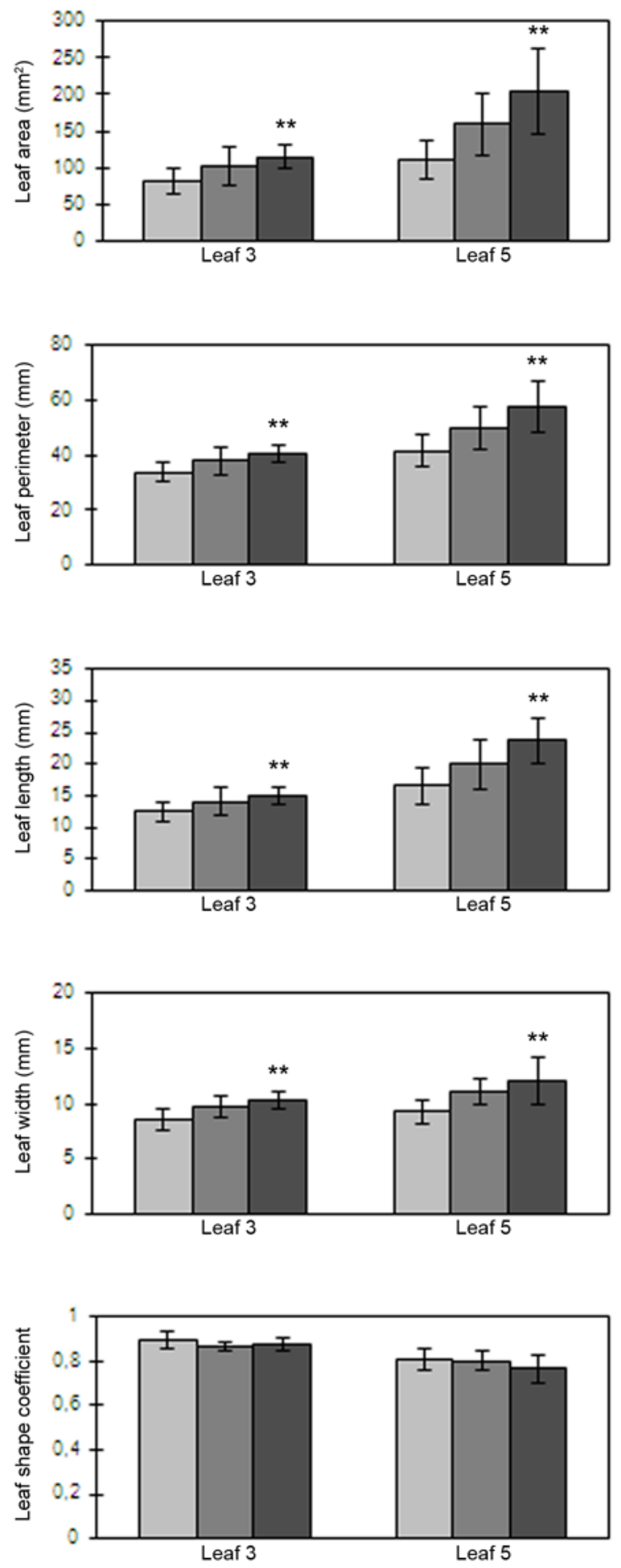

Fig. 4 Analysis of the morphology of leaves of WT (pale grey bars) and deg5 mutant plants (deg5-2, dark grey bars; deg5-1, black bars). Leaf area, perimeter, length, width and shape coefficient were measured for juvenile (leaf 3 ) and mature (leaf 5) leaves of WT as well as deg5-2 and deg5-1 mutant plants that had reached secondary stage 6.0. Asterisks indicate the data for which mutant/WT plants differences were significant $(P<0.01)$. Values are mean $\pm S D$.
To further understand the consequences of the loss of chloroplast protease AtDeg5 transmission electron microscopy was applied to investigate the ultrastructure of juvenile and mature leaves of both mutants and WT plants which reached secondary stage 6.0. Significant differences were found in the thylakoid system architecture, namely mature leaves (but not juvenile ones) of the mutants had more thylakoids stacked per granum and no intermutant differences were found to exist in this respect (Fig. 5); however, the thylakoid width was not altered in leaves of mutants vs WT plants. A striking difference was that juvenile leaves of deg5-2 mutant as well as both juvenile and mature leaves of deg5-1 showed an extensive transitory starch accumulation at the end of the night period while, according to the expectations, no starch grains were observed in chloroplasts of WT plants. To provide more detailed information about the impact of deficiency/loss of AtDeg5 on leaf transitory starch metabolism diurnal changes in contents of starch were measured in leaves of juvenile and mature leaves of both mutants and WT plants. It was found (Fig. 6) that the rate of accumulation of starch in the light in mature leaves of deg5-1 and juvenile leaves of both deg5-1 and deg5-2 mutants was higher than in WT plants and as result starch amount in mutant chloroplast reached significantly higher values at the end of the light period $(16 \mathrm{~h})$.

During the night the starch pool declined at approximately equal rates in both types of leaves of all three plant genotypes thus at the end of the dark period more unhydrolized starch was left in chloroplasts of mutant plants although the mutants were shown to be able to hydrolyze starch completely during an extended night. Thus the starch excess phenotype of the deg5 mutants seems to be caused by their ability to synthesize starch during light period at an elevated rate in particular leaves.

\section{$A / C_{i}$ responses of fully expanded leaves of WT and mutant plants}

To address the question why $\operatorname{deg} 5$ mutants synthesize excessive amounts of starch during the light period, $A / C_{i}$ responses were recorded at saturating irradiance for mature leaves of deg mutants and WT plants (no reliable measurements of photosynthetic gas exchange was possible with juvenile leaves due to their small area). These data allowed us to calculate the apparent maximal carboxylation rates limited by Rubisco amount, kinetics and activity $\left(V_{c, \max }\right)$ and maximal carboxylation rates limited by electron transport used in the regeneration of $\operatorname{RuBP}\left(J_{\max }\right)$. Mature leaves of WT plants and deg5 mutants exhibited very similar $A / C_{i}$ curve patterns and $V_{c, \max }$ and Jmax values were not statistically different among all three genotypes (Fig. 7).

Interpretation of mutant vs. WT plants differences in terms of AtDeg5 dosage effect and compensatory accumulation of AtDeg8

To facilitate interpretation of the mutants vs WT differences in terms of importance of AtDeg5 dosage and compensatory AtDeg8 accumulation, 33 phenotypic traits that are firmly established as AtDeg5-related since they are significantly altered significantly in at least deg5-1 (i.e. at least in the null mutant deg5-1) were classified into three groups according to similarity in the profile of the alterations (Tab. 1). The remaining traits, i.e. those not altered in any of 
(a)

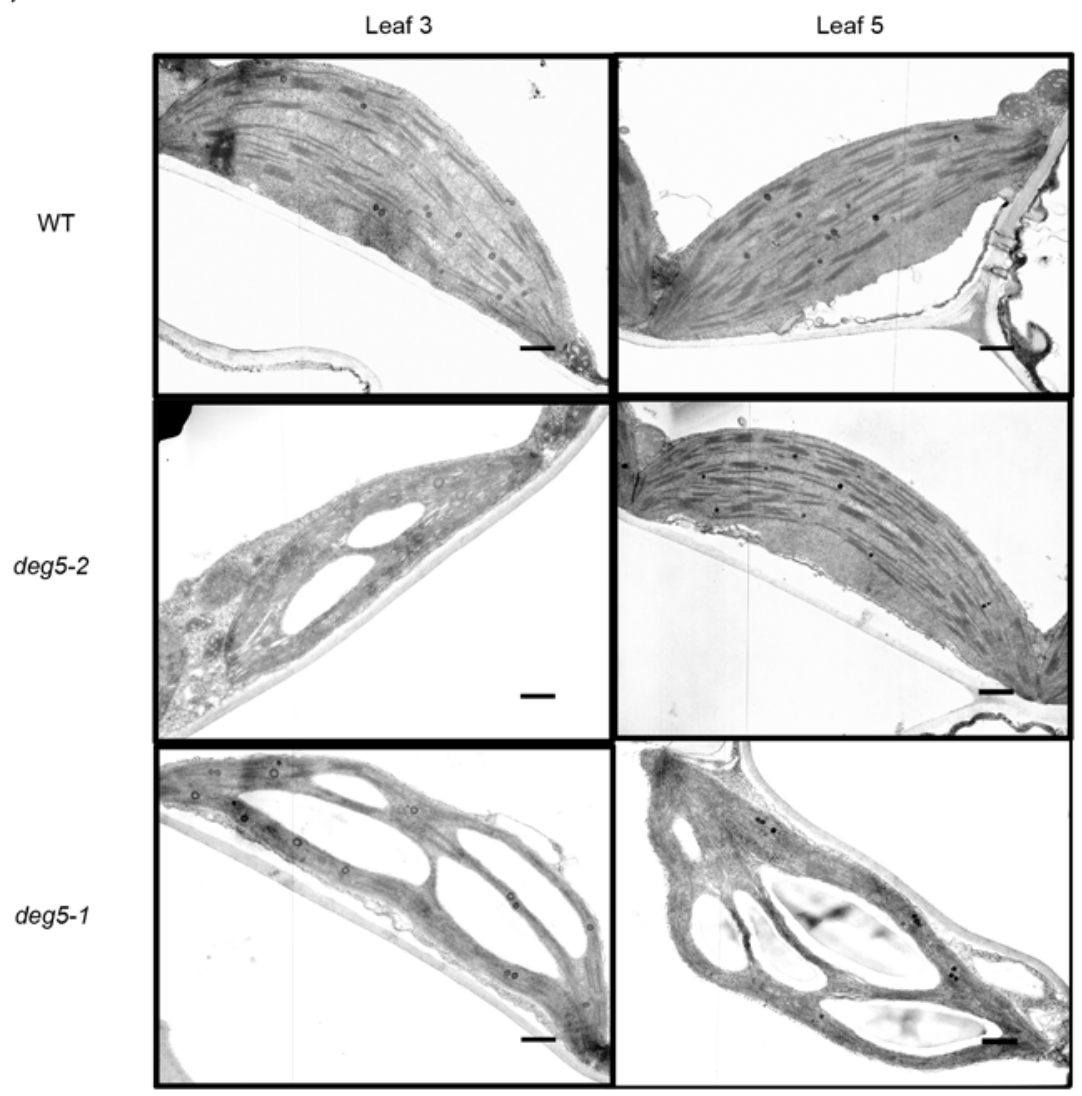

(b)
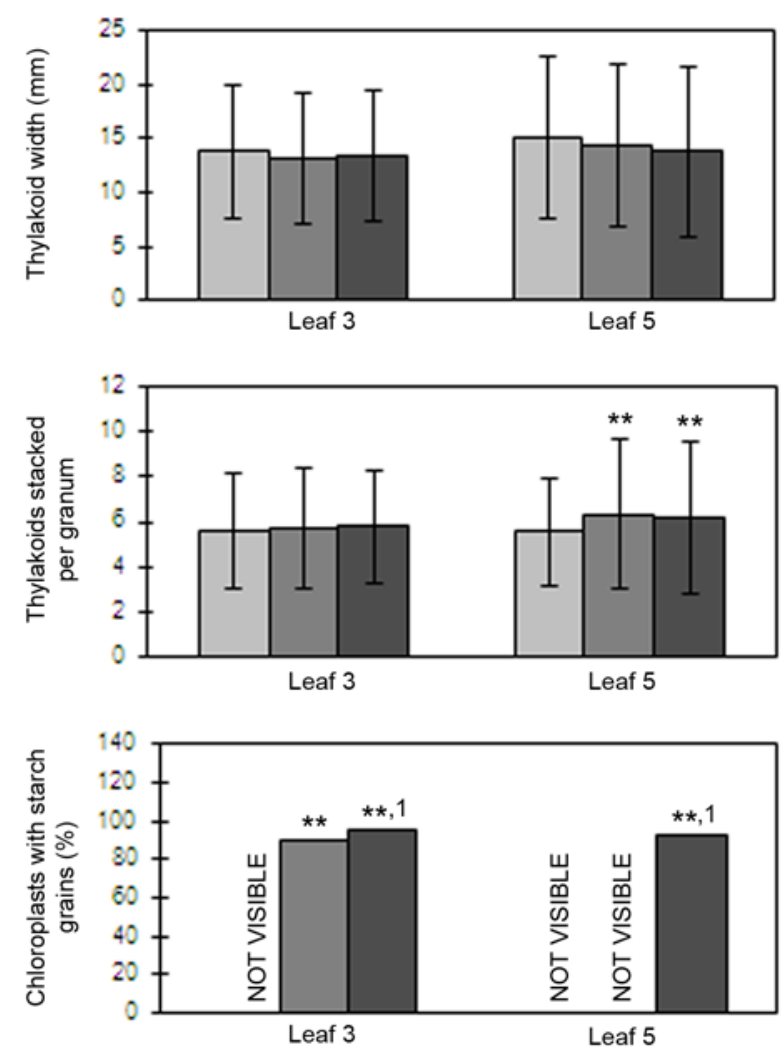

Fig. 5 Transmission electron microscopy analysis of chloroplast of WT (pale grey bars) and deg5 mutant plants (deg5-2, dark grey bars; deg5-1, black bars). a Electron micrographs of chloroplasts of juvenile (leaf 3) and mature (leaf 5) leaves of WT as well as deg5-2 and deg5-1 mutant plants that had reached secondary stage 6.0. Micrographs were taken at the end of the night period. Scale bar: $500 \mathrm{~nm}$. b Number of thylakoids stacked per granum, thylakoid width and frequency of chloroplasts with starch grains of juvenile (leaf 3 ) and mature (leaf 5) leaves of WT, deg5-1 and deg5-2 mutant plants which had reached secondary stage 6.0. Thylakoid width was measured as end-to-end distance between the two membranes of the same disk. Asterisks indicate the data for which mutant/WT plants differences were significant $(P<0.01)$ and 1 indicate the values for which deg5-1/deg5-2 differences were significant $(P<0.01)$. Values are mean $\pm S D$. 
Leaf 3

Leaf 5

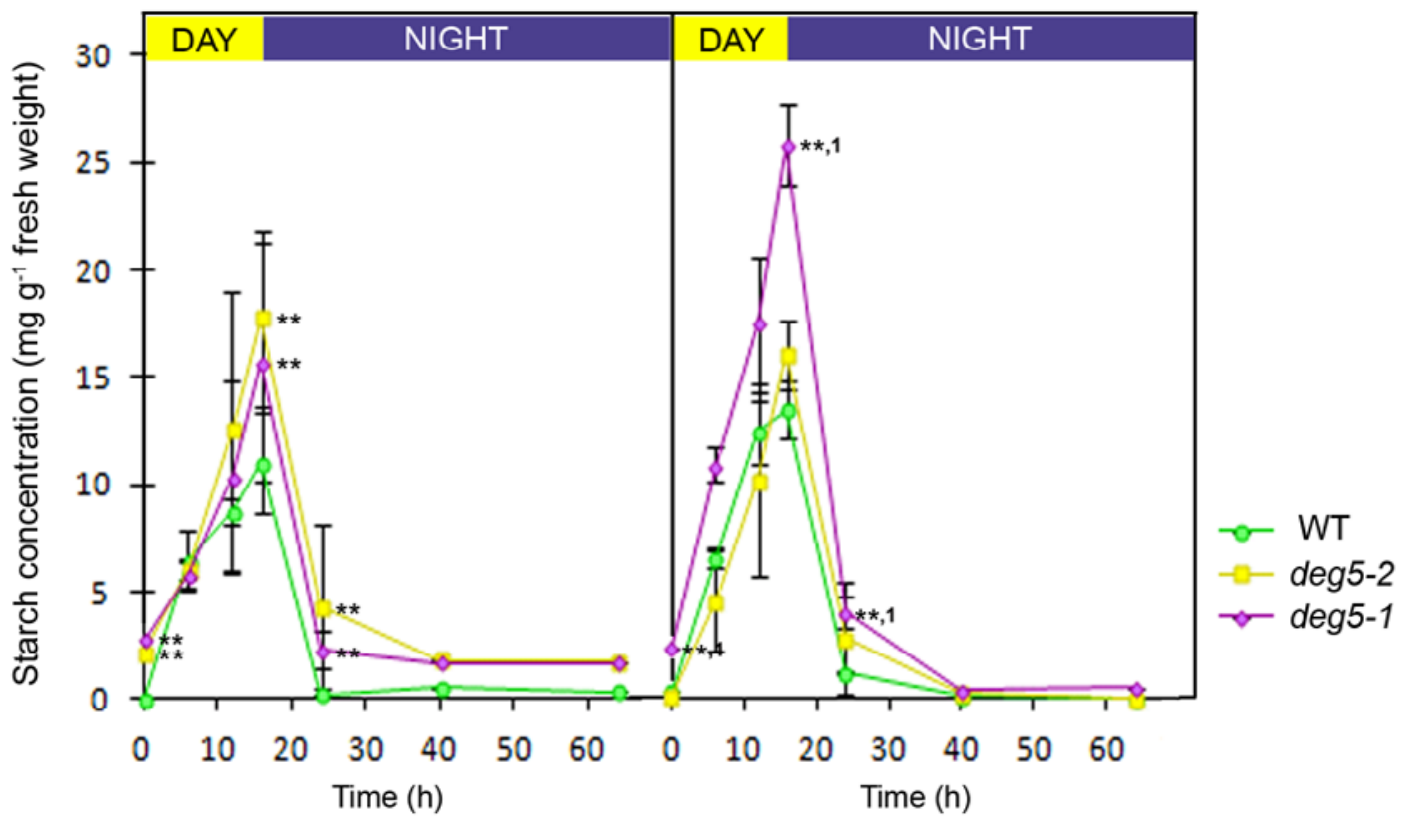

Fig. 6 Diurnal changes in starch content in leaves of WT and deg5 mutant plants. Starch content was determined in juvenile (leaf 3 ) and mature (leaf 5) leaves of WT as well as deg5-2 and deg5-1 mutant plants that had reached secondary stage 6.0. The measurements were performed over a 24-h photoperiod and an extended night. Asterisks indicate the data for which mutant/WT plants differences were significant $(P<0.01)$ and 1 indicate the values for which deg5-1/deg5-2 differences were significant $(P<0.01)$. Values are mean $\pm S D$. The absence of error bars means that they are smaller than the symbols.

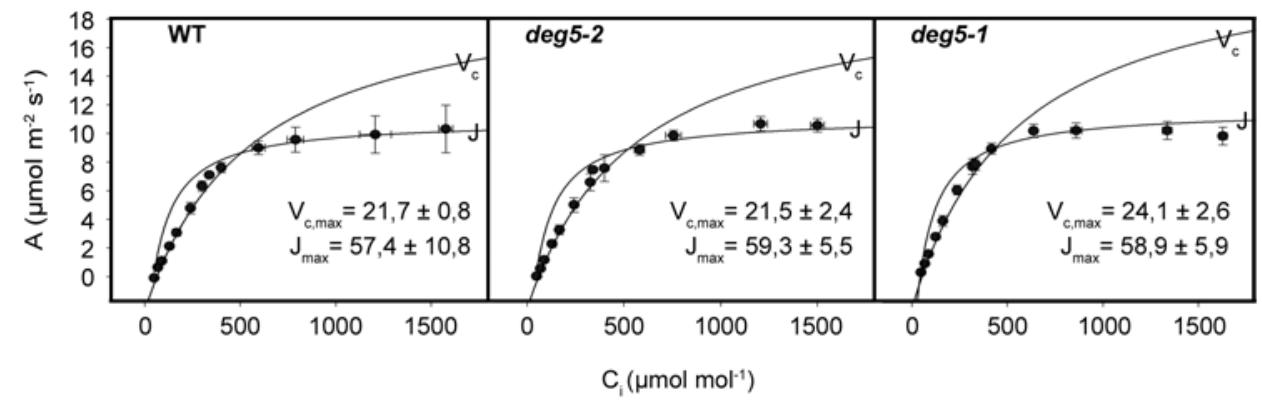

Fig. 7 Average $A / C_{i}$ curve for leaves of WT and $\operatorname{deg} 5$ mutant plants and Farquhar model fitted to the data. Closed circles represent measured rates of $\mathrm{CO}_{2}$ assimilation of mature (leaf 5) leaves $(A)$ whereas solid curves were obtained by fitting equations of the photosyntheis model [28] to experimental data. Datapoints corresponding to $C_{i}<400 \mu \mathrm{mol} \mathrm{mol}^{-1}$ were used to generate the curve for photosynthetis limited by Rubisco amount, kinetics and activity, whereas points corresponding to $C_{i}>400 \mu \mathrm{mol} \mathrm{mol}^{-1}$ were used to generate the curve for photosynthetis limited by rate of electron transport used for the regeneration of RuBP. Estimated apparent maximal Rubisco carboxylation rate $V_{c, \max }$ and maximal photosynthetic electron transport rate Jmax are shown in each panel. Values are mean $\pm S D$.

the two mutants are regarded either as unrelated to AtDeg5 or as AtDeg5-related but fully rescuable by AtDeg8 overdose.

For the prevailing majority of AtDeg5-dependent traits (26/33) belonged to groups 1 and 2 involving those for which no WT/deg5-2 alterations and significant WT/ deg5-1 alterations were detected, with (group 2) or without (group 1) significant between-mutants differences. It may be suspected that AtDeg8 is functionally interchangeable with AtDeg5 with regard to expression of these traits and AtDeg8 overaccumulation may rescue $23 \%$ of AtDeg 5 deficiency, but not AtDeg5 absence. As a consequence
AtDeg5 dosage determines whether intermutant differences are visible (group 2) or are not visible (group 1). The other possibility cannot be ruled out, however, that AtDeg8 is not able to rescue the loss of $23 \%$ of AtDeg 5 pool but AtDeg 5 has enough catalytic sites in excess for AtDeg5/AtDeg8 heterocomplex to function normally even when AtDeg 5 pool is reduced by $23 \%$ with regard to WT plants level. Depending on whether the number of AtDeg5 catalytic sites is substantially or only modestly excessive in relation to functional needs a phenotypic trait may fall into group 1 or 2 , respectively. 
Tab. 1 Phenotypic traits which are altered in $\operatorname{deg} 5$ mutants with regard to WT plants.

\begin{tabular}{|c|c|c|c|c|c|}
\hline \multirow{2}{*}{$\begin{array}{l}\text { Group } \\
\text { of traits }\end{array}$} & \multirow[b]{2}{*}{ Phenotypic trait } & \multicolumn{3}{|c|}{$\begin{array}{c}\text { Significance of WT/deg5-2, } \\
\text { WT/deg5-1 and deg5-1/deg5-2 } \\
\text { differences }\end{array}$} & \multirow[b]{2}{*}{ Interpretation } \\
\hline & & WT & $\operatorname{deg} 5-2$ & $\operatorname{deg} 5-1$ & \\
\hline 1 & $\begin{array}{l}\text { Secondary stage } 1.2 \\
\text { Secondary stage } 1.5 \\
\text { Secondary stage } 3.54 \\
\text { Secondary stage } 3.91 \\
\text { Secondary stage } 3.93 \\
\text { Secondary stage } 3.94 \\
\text { Secondary stage } 3.95 \\
\text { Secondary stage } 6.5 \\
\text { Secondary stage } 8.5 \\
\text { Area of leaf } 3 \\
\text { Area of leaf } 5 \\
\text { Perimeter of leaf } 3 \\
\text { Perimeter of leaf } 5 \\
\text { Length of leaf } 3 \\
\text { Length of leaf } 5 \\
\text { Width of leaf } 3 \\
\text { Width of leaf } 5\end{array}$ & 100 & 100 & $* *$ & $\begin{array}{l}\text { Chloroplast has a substantial excess } \\
\text { of AtDeg } 5 \text { catalytic sites with regard } \\
\text { to functional requirements or AtDeg8 } \\
\text { overdose is able to compensate for } 23 \% \\
\text { reduction of AtDeg } 5 \text { pool }\end{array}$ \\
\hline 2 & $\begin{array}{l}\text { Secondary stage } 3.16 \\
\text { Secondary stage } 3.58 \\
\text { Secondary stage } 3.59 \\
\text { Secondary stage } 3.96 \\
\text { Secondary stage } 3.97 \\
\text { Frequency of chloroplasts containing } \\
\text { starch grains in leaf } 5 \\
\text { Starch accumulation at } 0 \mathrm{~h} \text { in leaf } 5 \\
\text { Starch accumulation at } 16 \mathrm{~h} \text { in leaf } 5 \\
\text { Starch accumulation at } 24 \mathrm{~h} \text { in leaf } 5\end{array}$ & 100 & 100 & $* *, 1$ & $\begin{array}{l}\text { Chloroplast has a modest excess of } \\
\text { AtDeg } 5 \text { catalytic sites with regard to } \\
\text { functional requirements and AtDeg } 5 \\
\text { dosage effect is important or AtDeg } 8 \\
\text { overdose is able to compensate for } 23 \% \\
\text { reduction of AtDeg } 5 \text { pool }\end{array}$ \\
\hline 3 & $\begin{array}{l}\text { Frequency of chloroplasts containing } \\
\text { starch grains in leaf } 3 \\
\text { Thylakoids number per granum in leaf } 5 \\
\text { Starch accumulation at } 0 \mathrm{~h} \text { in leaf } 3 \\
\text { Starch accumulation at } 16 \mathrm{~h} \text { in leaf } 3 \\
\text { Starch accumulation at } 24 \mathrm{~h} \text { in leaf } 3 \\
\text { Seed length } \\
\text { Seed width }\end{array}$ & 100 & $* *$ & $* *$ & $\begin{array}{l}\text { Chloroplast does not have excess of } \\
\text { AtDeg } 5 \text { catalytic sites with regard to } \\
\text { functional requirements, no AtDeg } 5 \\
\text { dosage effect exists, AtDeg8 overdose is } \\
\text { not able to compensate for AtDeg } 5 \text { pool } \\
\text { reduction/absence }\end{array}$ \\
\hline
\end{tabular}

The traits have been selected so that they were altered significantly $(P<0.01)$ relative to WT plants (marked as 100) at least in deg5-1 mutant. The traits for which no significant deg5-2/WT differences were detected are marked as 100 . The asterisks indicate the data for which mutant/WT plants differences were significant $(P<0.01)$ and 1 indicates the values for which deg5-1/deg5-2 differences were significant $(P<0.01)$.

Markedly fewer phenotypic traits (7 out of 33) belonged to the group 3 including those for which significant WT vs. deg5-2 and WT vs. deg5-1 differences were detected, without significant deg5-2/deg5-1 differences. Such pattern was due to the absence of excessive amount of AtDeg 5 catalytic sites, lack of AtDeg5 dosage effect and inability of AtDeg8 to compensate AtDeg5 deficiency or absence.

\section{Discussion}

In this study we have shown that the presence of T-DNA insertion in the 4 th intron 872 bp downstream of ATG of At4g18370 gene (deg5-2 mutant, Fig. 1) results in lowering of AtDeg5 protein level to $77 \%$ of WT value. The deficiency or absence of AtDeg5 (deg5-1 null mutant) did not lead to changes in abundances of AtDeg1 and AtDeg2 proteins but the amount of AtDeg8 increased about twofold in both mutants (Fig. 2). Since AtDeg5 and AtDeg8 are thought to form a functional heterocomplex [14] a remarkable increase in AtDeg8 in both studied mutants may be regarded as a compensatory response and it could be inferred that AtDeg8 is functionally interchangeable with AtDeg5. The overdose of AtDeg8 could be substituting for the absent AtDeg5 in AtDeg5/AtDeg8 oligomeric heterocomplex. Consistent with this suggestion are the observations that subunits of other chloroplast proteolytic complexes are structurally interchangeable and functionally redundant, i.e. AtFtsH2/8 and AtFtsH1/5 protease pairs of AtFstH heterocomplex [35,36] and ClpR1 and ClpR3 subunits of Clp complex [37]. Alternatively, the excessive AtDeg8 pool could form functional oligomeric homocomplexes. AtDeg8 overdose could in fact substitute AtDeg5 in its catalytic functions as judged by the fact that for as many as 26 phenotypic traits that were altered 
in deg5-1 mutant no alteration was observable for the weak mutant (deg5-2) presumably due to ability of AtDeg8 to partially substitute for AtDeg5. Furthermore, the traits that were not altered in any of the mutants may be considered as AtDeg5-dependent/AtDeg8-rescuable although these traits may be just unrelated to both AtDeg5 and AtDeg8 as well. In any case, our data do not preclude the possibility that AtDeg8 may be functionally interchangeable with AtDeg5, at least with regard to regulation of some phenotypic traits (groups 1 and 2, Tab. 1).

Our results show that AtDeg5 is involved in leaf development, leaf growth, flower production and silique ripening as judged by a large array of examples of significant WT/deg5-1 delays in chronological progression of individual ontogenesis secondary stages (Fig. 3, Tab. 1). In contrast, deg5-2 mutant was not changed with regard to WT plants in this respect although intermutant differences have been identified with respect to five traits demonstrating the importance of AtDeg 5 dosage in these events (Tab. 1). Leaf production (development) and growth were shown to be controlled by genetic factors [38,39] linked to epidermal cell expansion and cell division with a key role of QTL at ERECTA marker in interaction with SNP295 and SNP21. Since functional relationships between these factors are complicated and far from clear it is not easy to imagine how AtDeg5 may influence these network. It is conceivable that the alterations in leaf growth identified in deg5-1 may be linked in a causal way to alterations in flower production related to AtDeg5 absence (Fig. 3) since epidermal cell area is controlled by mechanisms associated with the onset of flowering [40] with two highly conserved miRNA - miR156 and miR172 and their targets regarded as key components in reproductive phase change [41]. The final leaf area was demonstrated to be strongly correlated with epidermal cell number and epidermal cell area $[38,40]$ and AtDeg5 must be engaged in respective regulatory events since fully expanded leaves, both juvenile and mature ones were significantly affected in their morphological features as a result of AtDeg 5 repression (Fig. 4). It was shown earlier by us that a mixed population of mature leaves (7 and 8) of deg5-1 mutant had significantly increased area with regard to WT plants [19] thus our data strongly indicate that AtDeg5 is engaged in determining final leaf area of all types of rosette leaves.

Interestingly enough, mature leaves of mutant plants had more thylakoids stacked per granum than WT ones (6.2 vs. 5.6; Fig. 5), the effect described earlier for chloroplasts of juvenile leaves of $d e g 2$ mutants [16]. It may be suggested that early symptoms of senescence-related disintegration of thylakoid system occur in chloroplasts of WT plants which reach secondary stage 6.0 whereas in both $\operatorname{deg} 5$ mutants the symptoms are retarded. This notion is consistent with the

\section{Acknowledgments}

This work was supported by a Polish Ministry of Science and Higher Education in the form of statutory subsidy for the Department of Plant Physiology, Institute of Experimental Biology, Faculty of Biology, Adam Mickiewicz University, Poznań, Poland. We thank professor Jacek Oleksyn (Institute of Dendrology, Polish Academy of Sciences, Kórnik, Poland) for permission to use gas exchange system. appearance of other ultrastructural signs of AtDeg5-related retardation of early chloroplast senescence e.g. a reduction in cross-sectional area of plastoglobules with regard to WT plants [19]. It was suggested that senescence-related disintegration of thylakoid membranes may be controlled by chlorophyll $b$ reductase which paves the way for LHCII destabilization by starting off chlorophyll $b$ degradation [42]. However we did not find any differences in the steadystate level of LHCII apoproteins in fully expanded leaves of both deg5 mutants vs WT plants (data not shown) thus the reason why the mutants have more thylakoids per granum remains unclear.

Transmission electron microscopy showed an extensive transitory starch accumulation at the end of the night period in fully expanded juvenile leaves of deg5-2 mutant as well as both juvenile and mature leaves of deg5-1 (Fig. 6). It was demonstrated by enzymatic assays that during the light period the mutants accumulated more starch than WT plants (Fig. 6) but the three genotypes hydrolyzed starch during the night at approximately equal rates. Since carboxylation rates as well as electron transport rates were similar in mutants and WT plants (Fig. 7) it appears that AtDeg5 absence presumably results in an increase in the amount/activity of the enzyme(s) which operate(s) in starch biosynthesis pathway downstream of fructose-6-phosphate.

Although our results show that AtDeg5 is needed for normal plant growth and development, leaf morphology and chloroplast ultrastructure this protease does not seem to have any impact on the fitness of Arabidopsis, defined as seed production per plant, since both deg5 mutants did not differ from WT plants in this respect (data not shown).

One of two ways to globally interpret the heterogeneity of AtDeg5-regulated phenotypic traits (besides the involvement of AtDeg8 compensatory effect) is that the most numerous groups of traits which are altered in at least deg5-1 mutant (groups 1 and 2, Tab. 1) involve those for which AtDeg5 has a substantial (group 1) or a modest (group 2) excess of catalytic sites to function normally even when its pool is reduced by $23 \%$ with regard to WT plants whereas the group 3 involve the traits for which no AtDeg5 excess or dosage effect exists. The heterogeneity of AtDeg5-controlled phenotypic traits with regard to the degree of their dependence on AtDeg5 dosage/excess of catalytic sites would not be unexpected altogether since similar effects were described for other chloroplast proteases. It was demonstrated in this respect that var1-1 mutants - which are deficient in the chloroplast proteolytic AtFtsH heterocomplex - retain sufficient amount of AtFtsH catalytic sites for some processes to proceed normally (chloroplast biogenesis) but this amount is not sufficient for other processes to function normally (stressrelated degradation of Lhcb1-3 apoproteins) [23].

\section{Authors' contributions}

The following declarations about authors' contributions to the research have been made: designed the experiments: GJ, MB; performed the experiments: MB, TW; analyzed the experimental data: MB, TW, GJ; wrote the paper: GJ

\section{Competing interests}

No competing interests have been declared. 


\section{References}

1. Pesquet E. Plant proteases - from detection to function. Physiol Plant. 2012;145(1):1-4. http://dx.doi.org/10.1111/j.1399-3054.2012.01614.x

2. Lipińska B, Ang D, Georgopoulos C. Sequence analysis and transcriptional regulation of the Escherichia coli grpE gene, encoding a heat shock protein. Nucl Acids Res. 1988;16(15):7545-7562. http:// dx.doi.org/10.1093/nar/16.15.7545

3. Strauch KL, Beckwitt J. An Escherichia coli mutation preventing degradation of abnormal periplasmic proteins. Proc Natl Acad Sci USA. 1988;85(5):1576-1580. http://dx.doi.org/10.1073/pnas.85.5.1576

4. Wilken C, Kitzing K, Kurzbauer R, Ehrmann M, Clausen T. Crystal structure of the DegS stress sensor: how a PDZ domain recognizes misfolded protein and activates a protease. Cell. 2004;117(4):483-494. http://dx.doi.org/10.1016/S0092-8674(04)00454-4

5. Jiang J, Zhang X, Chen Y, Wu Y, Zhou ZH, Chang Z, Sui SF. Activation of DegP chaperone-protease via formation of large cagelike oligomers upon binding to substrate proteins. Proc Natl Acad Sci USA. 2008;105(33):11939-11944. http://dx.doi.org/10.1073/ pnas.0805464105

6. Bai XC, Pan XI, Wang XJ, Ye YY, Chang LF, Leng D, et al. Characterization of the structure and function of Escherichia coli DegQ as a representative of the DegQ-like proteases of bacterial HtrA family proteins. Structure. 2011;19(9):1328-1337. http://dx.doi.org/10.1016/j. str.2011.06.013

7. Ortega J, Iwańczyk J, Jomaa A. Escherichia coli DegP: a structure-driven functional model. J Bacteriol. 2009;191(15):4705-4713. http://dx.doi. org/10.1128/JB.00472-09

8. Schuhmann H, Huesgen PF, Adamska I. The family of Deg/HtrA proteases in plants. BMC Plant Biol. 2012;12(1):1-14. http://dx.doi. org/10.1186/1471-2229-12-52

9. Schuhmann H, Adamska I. Deg proteases and their role in protein quality control and processing in different subcellular compartments of the plant cell. Physiol Plant. 2012;145(1):224-234. http://dx.doi. org/10.1111/j.1399-3054.2011.01533.x

10. Schuhmann H, Mogg U, Adamska I. A new principle of oligomerization of plant DEG7 protease based on interactions of degenerated protease domains. Biochem J. 2011;435(1):167-174. http://dx.doi. org/10.1042/BJ20101613

11. Kley J, Schmidt B, Boyanov B, Stolt-Bergner PC, Kirk R, Ehrmann $\mathrm{M}$, et al. Structural adaptation of the plant protease Deg1 to repair photosystem II during light exposure. Nat Struct Mol Biol. 2011;18(6):728-731. http://dx.doi.org/10.1038/nsmb.2055

12. Sun XW, Fu TJ, Chen N, Guo JK, Ma JF, Zou MJ, et al. The stromal chloroplast Deg7 protease participates in the repair of photosystem II after photoinhibition in Arabidopsis. Plant Physiol. 2010;152(3):12631273. http://dx.doi.org/10.1104/pp.109.150722

13. Sun W, Gao F, Fan H, Shan X, Sun R, Liu L, et al. The structures of Arabidopsis Deg5 and Deg8 reveal new insights into HtrA proteases. Acta Crystallogr D Biol Crystallogr. 2013;69(5):830-837. http://dx.doi. org/10.1107/S0907444913002023

14. Sun XW, Peng L, Guo J, Chi W, Ma J, Lu C, et al. Formation of DEG5 and DEG8 and their involvement in the degradation of photodamaged photosystem II reaction center D1 protein in Arabidopsis. Plant Cell. 2007;19(4):1347-1361. http://dx.doi.org/10.1105/tpc.106.049510

15. Kapri-Pardes E, Naveh L, Adam Z. The thylakoid lumen protease Deg1 is involved in the repair of photosystem II from photoinhibition in Arabidopsis. Plant Cell. 2007;19(3):1039-1047. http://dx.doi. org/10.1105/tpc.106.046573

16. Luciński R, Misztal L, Samardakiewicz S, Jackowski G. The thylakoid protease Deg2 is involved in stress-related degradation of the photosystem II light-harvesting protein Lhcb6 in Arabidopsis thaliana. New Phytol. 2011;192(1):174-186. http://dx.doi. org/10.1111/j.1469-8137.2011.03782.x

17. Kato Y, Sun X, Zhang L, Sakamoto W. Cooperative D1 degradation in the photosystem II repair mediated by chloroplastic proteases in Arabidopsis. Plant Physiol. 2012;159(4):1428-1439. http://dx.doi. org/10.1104/pp.112.199042
18. Zienkiewicz M, Ferenc A, Wasilewska W, Romanowska E. High light stimulates Deg1-dependent cleavage of the minor LHCII antenna proteins CP26 and CP29 and the PsbS protein in Arabidopsis thaliana. Planta. 2012235(2):279-288. http://dx.doi.org/10.1007/ s00425-011-1505-X

19. Luciński R, Misztal L, Samardakiewicz S, Jackowski G. Involvement of Deg5 protease in wounding-related disposal of PsbF apoprotein. Plant Physiol Biochem. 2011;49(3):311-320. http://dx.doi.org/10.1016/j. plaphy.2011.01.001

20. Murashige T, Skoog F. A revised medium for rapid growth and bio assays with tobacco tissue cultures. Physiol Plant. 1962;15(3):473-497. http://dx.doi.org/10.1111/j.1399-3054.1962.tb08052.x

21. Grabsztunowicz M, Jackowski G. Isolation of intact and pure chloroplasts from leaves of Arabidopsis thaliana plants acclimated to low irradiance for studies on Rubisco regulation. Acta Soc Bot Pol. 2013;82(1):91-95. http://dx.doi.org/10.5586/asbp.2012.043

22. Laemmli UK. Cleavage of structural proteins during the assembly of the head of bacteriophage T4. Nature. 1970;227(5259):680-685. http://dx.doi.org/10.1038/227680a0

23. Luciński R, Jackowski G. AtFtsH heterocomplex-mediated degradation of apoproteins of the major light harvesting complex of photosystem II (LHCII) in response to stresses. J Plant Physiol. 2013;170(12):10821089. http://dx.doi.org/10.1016/j.jplph.2013.03.008

24. Boyes DC, Zayed AM, Ascenzi R, McCaskill AJ, Hoffman NE, Davis KR, et al. Growth stage-based phenotypic analysis of Arabidopsis: a model for high throughput functional genomics in plants. Plant Cell. 2001;13(7):1499-1510. http://dx.doi.org/10.2307/3871382

25. Kincaid DT, Schneider RB. Quantification of leaf shape with a microcomputer and Fourier transform. Can J Bot. 1983;61(9):2333-2342. http://dx.doi.org/10.1139/b83-256

26. Western TL, Skinner DJ, Haughn GW. Differentiation of mucilage secretory cells of the Arabidopsis seed coat. Plant Physiol. 2000;122(2):345-355. http://dx.doi.org/10.1104/pp.122.2.345

27. Chow PS, Landhausser SM. A method for routine measurements of total sugar and starch content in woody plant tissues. Tree Physiol. 2004;24(10):1129-1136. http://dx.doi.org/10.1093/treephys/24.10.1129

28. Farquhar GD, von Caemmerer S, Berry JA. A biochemical-model of photosynthetic $\mathrm{CO}_{2}$ assimilation in leaves of $\mathrm{C} 3$ species. Planta. 1980;149(1):78-90. http://dx.doi.org/10.1007/BF00386231

29. Tanaka Y, Sugano SS, Shimada T, Nishimura I. Enhancement of leaf photosynthetic capacity through increased stomatal density in Arabidopsis. New Phytol. 2013;198(3):757-764. http://dx.doi. org/10.1111/nph.12186

30. Long SP, Bernacchi CJ. Gas exchange measurements, what can they tell us about the underlying limitations to photosynthesis? Procedures and sources of error. J Exp Bot. 2003;54(392):2393-2401. http://dx.doi. org/10.1093/jxb/erg262

31. Sharkey TD, Bernacchi CJ, Farquhar GD, Singsaas EL. Fitting photosynthetic carbon dioxide response curves for C3 leaves. Plant Cell Environ. 2007;30(9):1035-1040. http://dx.doi. org/10.1111/j.1365-3040.2007.01710.x

32. Arnon D. Copper enzymes in isolated chloroplasts. Polyphenol oxidase in Beta vulgaris. Plant Physiol. 1949;24(1):1-15. http://dx.doi. org/10.1104/pp.24.1.1

33. Lancashire PD, Bleiholder $H$, van der Boom T, Langeluddeke $P$, Stauss R, Weber E, et al. A uniform decimal code for growth stages of crops and weeds. Ann Appl Biol. 1991;119(3):561-601. http://dx.doi. org/10.1111/j.1744-7348.1991.tb04895.x

34. Telfer A, Bollman KM, Poethig RS. Phase change and the regulation of trichome distribution in Arabidopsis thaliana. Development. 1997;124(3):645-654.

35. Yu F, Park S, Rodermel SR. The Arabidopsis FtsH metalloprotease gene family: interchangeability of subunits in chloroplast oligomeric complexes. Plant J. 2004;37(6):864-876. http://dx.doi. org/10.1111/j.1365-313X.2003.02014.x

36. Yu F, Park S, Rodermel SR. Functional redundancy of AtFtsH 
metalloproteases in thylakoid membrane complexes. Plant Physiol. 2005;138(4):1957-1966. http://dx.doi.org/10.1104/pp.105.061234

37. Stanne TM, Sjögren LL, Koussevitzky S, Clarke AK. Identification of new protein substrates for the chloroplast ATP-dependent Clp protease supports its constitutive role in Arabidopsis. Biochem J. 2009;417(1):257-268. http://dx.doi.org/10.1042/BJ20081146

38. Tisné S, Reymond M, Vile D, Fabre J, Dauzat M, Koornneef M, et al. Combined genetic and modeling approaches reveal that epidermal cell area and number in leaves are controlled by leaf and plant developmental processes in Arabidopsis. Plant Physiol. 2008;148(2):1117-1127. http://dx.doi.org/10.1104/pp.108.124271

39. Massonnet C, Vile D, Fabre J, Hannah MA, Caldana C, Lisec J, et al. Probing the reproducibility of leaf growth and molecular phenotypes: a comparison of three Arabidopsis accessions cultivated in ten laboratories. Plant Physiol. 2010;152(4):2142-2157. http://dx.doi. org/10.1104/pp.109.148338

40. Cookson SJ, Chenu K, Granier C. Day length affects the dynamics of leaf expansion and cellular development in Arabidopsis thaliana partially through floral transition timing. Ann Bot. 2007;99(4):703-711. http://dx.doi.org/10.1093/aob/mcm005

41. Huijser P, Schmid M. The control of developmental phase transitions in plants. Development. 2011;138(19):4117-4129. http://dx.doi. org/10.1242/dev.063511

42. Kusaba M, Ito H, Morita R, Iida S, Sato Y, Fujimoto M, et al. Rice NON-YELLOW COLORING1 is involved in light-harvesting complex II and grana degradation during leaf senescence. Plant Cell. 2007;19(4):1362-1375. http://dx.doi.org/10.1105/tpc.106.042911 